\title{
Le Manifeste scalène de la « quatrième génération » d'artistes en Acadie : vers une relève régionale
}

\author{
Pénélope Cormier
}

Université de Moncton, campus d'Edmundston

Lors d'une conférence prononcée en 2014, Herménégilde Chiasson disait observer une nouvelle étape de l'évolution de l'art acadien : «Il y a désormais en Acadie, avance-t-il, quatre générations d'artistes que le public peut situer sans mal dans l'histoire de l'art, puisque leurs pratiques respectives s'inscrivent dans des mouvements artistiques distincts. » (Chiasson, 2017, p. 111) S'il est pertinent de commencer une réflexion sur la relève littéraire acadienne avec Chiasson, c'est que son ombre plane doublement sur elle : en plus d'être l'artiste acadien le plus en vue présentement, donc celui contre lequel une relève doit 
nécessairement se positionner et se définir, il est aussi l'un des commentateurs les plus assidus de l'histoire récente de l'art en Acadie. Or, il est à la fois juge et partie dans celle-ci, s'inscrivant dans la deuxième génération d'artistes acadiens selon le modèle qu'il propose, et on sait qu'il a été très critique des propositions de la collectivité d'artistes qui l'a immédiatement suivi (voir Boudreau, 2004 et Cormier, 2009). C'est donc une nuance majeure qu'il apporte à son propos en reconnaissant la poursuite de l'histoire artistique acadienne, par la prise en compte d'une prochaine relève.

L’importance du moment se mesure également à la cohésion et à la cohérence que se donnent eux-mêmes les artistes de cette relève, en contraste avec la « troisième génération », à laquelle on a donné le nom d' «école Aberdeen »(Cossette et Laparra, 2001; Cormier, 2012; Laparra, 2004), qui fonctionnait surtout comme réseau institutionnel aux liens très relâchés. Dans un article la décrivant, je notais que « [n]i cercle littéraire structuré ni avantgarde organisée, l'école Aberdeen n'a pas de programme esthétique officiel, voire informel » (Cormier, 2012, p. 180). Cette fois, certains représentants de la relève littéraire acadienne proposent une sorte de manifeste, sous forme d'un spectacle de poésie : le Manifeste scalène de Sébastien Bérubé, Gabriel Robichaud et Jonathan Roy, présenté pour la première fois en mars 2016 à Caraquet, dans le cadre du Festival acadien de poésie. Durant deux années, il a été présenté dans différents cadres, dont deux fois à Québec (en mars et en octobre 2017), deux fois à Edmundston (en octobre 2017 et en avril 2018) et une fois à Moncton (en avril 2018), avant de revenir à Caraquet pour une ultime représentation en avril $2018^{1}$.

\footnotetext{
${ }^{1}$ Au fil des représentations, le texte du spectacle a connu certaines modifications. La version utilisée ici correspond à l'état du texte à l'automne 2017.
} 
PÉNÉLOPE CORMIER, « Le Manifeste scalène de la “ quatrième génération ” d'artiste en Acadie : vers une relève régionale »

La description de l'événement revendique explicitement l'appartenance des créateurs à une nouvelle étape de l'histoire de la littérature acadienne. Certes, ils y revendiquent une appartenance générationnelle, mais c'est leur insistance sur la représentativité régionale de l'Acadie du Nouveau-Brunswick de leur spectacle qui est réellement originale dans le paysage littéraire acadien :

MANIFESTE SCALÈNE, c'est la rencontre sur scène de trois poètes de la nouvelle génération, issus du triangle scalène des trois territoires de l'Acadie du N.-B. Au cœur de la rencontre, trois textes construits sur la répétition, l'anaphore, et sur le ton du manifeste. (Bérubé, Robichaud et Roy, 2017, p. 2)

Le spectacle est constitué du tressage des poèmes « Hymne » de Sébastien Bérubé (2017, p. 26-52), «Manifeste diasporeux » de Jean-Philippe Raîche et Gabriel Robichaud (dans Robichaud, 2018, p. 157-165), ainsi que « voix rurale » de Jonathan Roy (2012, p. 44-47, et 2014 , s. p.), dont une version augmentée a été publiée dans le recueil savèches à fragmentation (Roy, 2019, p. 116-125). Comportant 535 vers qui sont lus - sauf quelques exceptions qui seront examinées plus loin - par leur auteur respectif, le spectacle dure une vingtaine de minutes; la plupart des représentations y intercalent cependant une lecture solo de chaque poète, amenant la durée de l'activité jusqu'à un peu moins d'une heure.

Bien que ses auteurs ne cherchent pas à faire du Manifeste scalène le programme de toute une génération, le spectacle est porteur d'un propos collectif dont il importe de cerner les enjeux, qui surgissent également dans les œuvres individuelles des poètes. Malgré ce que laisse entendre le premier mot de son titre, le spectacle de Bérubé, Robichaud et Roy n'a pas l'ambition de solliciter l'histoire des ruptures esthétiques tranchées, érudites et militantes des manifestes de la francophonie littéraire, de La Deffence et Illustration de la Langue Francoyse (1549) jusqu'à Pour une littérature-monde en français (2007), en passant par le 
Manifeste du surréalisme (1924). Entre autres par sa qualité d'art poétique plutôt que de texte programmatique, le Manifeste scalène s'éloigne assurément de cette logique de rupture. Les auteurs s'en servent même en bonne partie pour s'inscrire dans la continuité historique de la littérature acadienne. La principale rupture revendiquée par le spectacle se réalise moins dans le temps - entre les générations artistiques - que dans l'espace, par sa prise en compte de l'éparpillement géographique actuel de la vie littéraire acadienne. On le voit dès le second terme du titre : le triangle évoqué correspond aux trois régions de l'Acadie du NouveauBrunswick, auxquelles s'identifient les trois poètes : le Nord-Est pour Roy, le Nord-Ouest pour Bérubé et le Sud-Est pour Robichaud. Il s'agit ici de revenir sur la portée artistique de cette proposition spatiale, en postulant l'émergence d'une littérature acadienne ancrée non plus seulement à Moncton, mais dans le nord du Nouveau-Brunswick.

Pour comprendre ce phénomène, il faut voir qu'il fait suite au mouvement principal de la littérature acadienne depuis les années 1970 jusqu'au milieu des années 2000, qui a été d'investir l'espace urbain, faisant de la ville de Moncton, comme l'analyse Ariane Brun del Re, « la capitale d'une littérature acadienne moderne » $(2012, \text { p. 17) })^{2}$. Cette réalisation, à la fois institutionnelle et discursive, sur laquelle le poète Gérald Leblanc, entre autres, a misé sa carrière (Boudreau, 2007; Brun del Re, 2012), a permis à Moncton de devenir un pôle d'attraction littéraire, notamment pour les poètes qui ont formé l'école Aberdeen. Rapidement, dès le début des années 2000, on voit par contre qu’une première «sortie de Moncton» est effectuée par les artistes de la Baie Sainte-Marie en Nouvelle-Écosse, réagissant à la centralité de la ville par une affirmation artistique rurale. Il me semble que les

\footnotetext{
${ }^{2}$ Voir aussi Boudreau, 2007; Boudreau et White, 2009; Bruce, 2005; Doyon-Gosselin, 2015.
} 
PÉNÉLOPE CORMIER, «Le Manifeste scalène de la “ quatrième génération " d'artiste en Acadie : vers une relève régionale »

artistes du nord du Nouveau-Brunswick, de la Péninsule acadienne au Madawaska, sont en train de prendre le relais de cette régionalisation de la littérature acadienne, et le Manifeste scalène en est l'une des manifestations les plus concrètes. Avant tout, il convient cependant de revenir sur comment on a construit la catégorie de « relève » et sur quelles bases on en a défini les enjeux dans le discours sur la littérature acadienne.

\section{Détracteurs et défenseurs de la « relève »}

Parler de la relève littéraire provoque inévitablement, en Acadie comme ailleurs, des débats sur sa qualité ou son indigence, qui font bien entendu partie du processus de reconnaissance des nouvelles plumes. Cependant, en raison peut-être de la quasi absence d'espace médiatique de discours et d'échange sur l'actualité artistique en Acadie (Brun del Re et Cormier, 2015), peu de traces écrites demeurent de ces débats qui revêtent en définitive un caractère informel, ou partiel lorsque des textes écrits renvoient à des ouï-dire. Par exemple, en 2005, Benoit Doyon-Gosselin commence sa critique de quelques recueils de poésie publiés au début des années 2000, intitulée « Y a-t-il une relève en poésie acadienne? », par le constat suivant : «À en croire certains bien-pensants de l'institution littéraire acadienne, la relève en poésie acadienne n'existerait tout simplement pas. » (Doyon-Gosselin, 2005, p. 38) Plus récemment, Mathieu Wade indiquait laconiquement que le recueil Acadie Road (2018) de Gabriel Robichaud « a connu un réel succès critique et commercial » $(2019$, s. p.) et entreprenait, en réaction, d'en recenser les failles. Certes, ce texte publié dans le webzine Astheure réagissait à l'attribution du Prix Champlain au recueil, voire à son accueil favorable au Québec; n'empêche qu'en Acadie, la seule autre critique à paraître sur Acadie Road, celle d'Isabelle Kirouac-Massicotte également sur Astheure, relevait déjà en 2018 les mêmes 
points faibles du recueil, quoique sur un autre ton. Ces deux textes, bien que n'étant pas en dialogue, constituent l'essentiel de la réception acadienne du recueil.

En plus de réagir à leur interprétation subjective d'un «air du temps », les commentateurs de la relève, détracteurs comme défenseurs, sont souvent convaincus d'être dans la minorité et leurs positions n'en sont souvent que plus tranchées, comme le montre certainement le texte de Wade. Quoiqu'il en soit, le débat sur le statut incertain des nouvelles plumes se cristallise toujours autour du fait que l'un des problèmes de reconnaissance de toute « relève » est que, dans les esprits, le passage des générations littéraires doit se faire sur le mode de la rupture, spontanée et absolue, avec ce qui précède. Les nouveaux arrivants sur la scène littéraire sont jugés à l'étalon de cette valeur pourtant contestable. Car il faut bien reconnaître, à la suite de Boudreau, qu'il est impossible, dans les années 1990, 2000 et maintenant 2010, d'effectuer le passage des générations en littérature acadienne sur le modèle du conflit entre les Anciens et les Modernes : «Ce qui est nouveau cependant et qui témoigne de l'évolution de l'institution littéraire acadienne, c'est que l'opposition ne se fait plus entre tradition et modernité, mais entre deux formes d'avant-garde » (Boudreau, 2004, p. 191). En Acadie, la tendance des artistes de la relève des années 1990 et 2000 à s'inscrire en continuité, plutôt qu'en rupture, de la génération précédente est remarquée : « ils s'insèrent dans un réseau social spécifique; c'est là une filiation dont sont très conscients ces jeunes auteurs, qui dédient des poèmes à Herménégilde Chiasson, à Guy Arsenault et à Gérald Leblanc. » (McLaughlin, 2001, p. 137) Ici, la particularité est donc que la rupture intergénérationnelle est réclamée et définie en amont par l'artiste établi, en l'occurrence Chiasson, un des seuls détracteurs à avoir désavoué publiquement cette relève et, surtout, son absence de rupture : « J'ai souvent dit que s'il fallait un père à tuer j'étais prêt à jouer ce rôle car je crois que ce 
PÉNÉLOPE CORMIER, «Le Manifeste scalène de la “ quatrième génération " d'artiste en Acadie : vers une relève régionale »

conflit et ce respect sont essentiels à l'affirmation d'une vision et d'un renouvellement de la pensée » (2005, p. 18-19).

Dans le portrait qu'il brosse de l'évolution de l'art en Acadie en trois - et tout récemment quatre - générations, l'adhésion à ce régime moderniste de l'art, qui appelle une fuite constante vers l'avant, à coup de ruptures esthétiques, est centrale. Il fait même d'une vaste entreprise de rattrapage de la modernité l'acte fondateur de toute sa génération (contre celle d'Antonine Maillet),

pour signaler au monde entier que nous aussi nous étions au courant des repères qui nous permettaient de rejoindre le siècle. Milan Kundera, Joseph Beuys, Jean-Luc Goddard ou Bob Wilson devenaient des références plus pertinentes que la poutine râpée, l'accent de Bouctouche, les tapis hookés ou le bateau fantôme. (1998a, s. p.)

Si la transition entre la première et la deuxième génération artistique en Acadie est ainsi commodément associée à la division entre tradition et modernité, la transition suivante, entre les générations «modernes » de Chiasson (et ses contemporains) et de l'école Aberdeen, nécessitera une gymnastique discursive.

Il reproche aux auteurs qui le suivent de fraterniser avec l'anglais, ce qui se matérialise dans l'écriture par la revendication du chiac (le vernaculaire français de la région de Moncton accueillant des emprunts phonétiques et morphologiques à l'anglais) comme emblème identitaire. Alors que d'aucuns ont replacé ce phénomène dans le mouvement planétaire de valorisation de l'hybridité linguistique et culturelle, et donc à l'avant-garde de la production artistique (voir Leclerc, 2010 et McLaughlin, 2013), Chiasson y voit un anachronisme, c’est-à-dire un retour à la tradition : « avec ses particularismes, le chiac est en voie de remplacer la langue vernaculaire, telle qu'on la retrouve chez Maillet par exemple, également marquée par des particularismes mais d'un tout autre ordre. » $(2017$, p. 230) 
Autrement dit, l'évolution de l'art en Acadie se fait pour lui non pas en suivant la ligne de temps de la modernité artistique, où l'avenir est porteur de progrès, mais par un mouvement cyclique, « incessant zig zag entre tradition et modernité » (Chiasson, 2005, p. 20).

En plus de cette logique temporelle de l'évolution des arts en Acadie, Chiasson lui reconnaît une logique spatiale, se présentant comme membre de «la première génération à avoir refusé l'exil et à penser qu'ensemble nous pouvions peut-être aménager un lieu, une Acadie, dont on nous dit qu'elle ne nous appartient plus et qu'elle glissera un jour dans l'océan d'une vaste et insondable diaspora. » (1994, p. 323) Il sera même l'un des plus virulents participants au débat sur l'exil des artistes acadiens qui enflamma le milieu artistique acadien pour toute la première moitié des années 1990 (voir Gauvin, 2004). Mais sur ce sujet, l'école Aberdeen est inattaquable, étant résolument établie en Acadie; Chiasson leur reprochera cependant, en plus de leur ouverture à l'anglais, leur désengagement social, c'est-à-dire de «faire absence de l'espace public pour se porter vers l'espace intérieur » (2006, s. p.). Cette fois, la figure du cycle est porteuse d'espoir pour l'essayiste : « il est à prévoir, dit-il dans une conférence en 2006, dans cette alternance des courants, si l'on en croit l'idée que l'histoire progresse en spirale, qu'il y aura bientôt un retour à l'idée d'une consolidation des frontières qui revisitera le concept de l'acadianité et de ses implications territoriales. » (s. p.) Ce moment lui semble arrivé, comme en témoigne son enthousiasme, une dizaine d'années plus tard, à reconnaître une «quatrième génération » d'artistes acadiens; on comprend aussi que l'intérêt de Chiasson pour cette « nouvelle relève » porte en bonne partie sur son engagement territorial. 
PÉNÉLOPE CORMIER, «Le Manifeste scalène de la “ quatrième génération " d'artiste en Acadie : vers une relève régionale »

\section{Le «manifeste Moncton » et quelques réserves rurales}

L'entreprise conjointement esthétique et idéologique de Chiasson, qu'il présente comme le fait de sa génération, conjugue également la logique temporelle et la logique spatiale de l'évolution de la littérature acadienne : « il nous restait comme projet, écrit-il, de réconcilier Acadie et modernité, un travail surhumain, une contradiction aussi nébuleuse que les termes qui la compose »(Chiasson, 1998b, p. 81). L'espace acadien à imprégner de modernité artistique est pour lui l'Acadie prise en son ensemble, comme espace « national ». Pourtant, sur une trentaine d'années, on a plutôt associé, en littérature acadienne, la modernité artistique à l'espace urbain, c'est-à-dire à la ville de Moncton.

C'est comme si, suivant la pique de Jean-Guy Pilon dans la revue québécoise Liberté en 1969, et maintes fois citée depuis, où il remarquait que «Moncton est une ville laide qui doit sûrement être l'œuvre de quelqu'un » (p. 155), les artistes acadiens ont entrepris, sous le leadership du poète Gérald Leblanc, de faire de Moncton leur œuvre. La première opération, comme le note Clint Bruce, consiste à faire de Moncton un espace acadien, ou à y négocier un espace acadien :

Dans un Moncton majoritairement anglophone et d'emblée hostile au projet de société des Acadiens, la solution, paraît-il, doit dépasser la sphère politique, car si, historiquement, la modernité implique une existence urbaine, il faut bien sûr pouvoir faire réellement sien le lieu où s'élabore cette modernité. En littérature, c'est Gérald Leblanc qui s'est attaché à définir cette Acadie proprement citadine. (2005, p. 206)

À sa suite, d'autres artistes acadiens revendiqueront Moncton comme décor de la modernité artistique acadienne et, en tant que telle, elle devient rapidement, et durablement, un pôle d'attraction pour les artistes de partout en Acadie : «Moncton est une ville qui attire, une 
ville qui présente un appel de l'avenir, qui offre la possibilité d'un changement radical : la modernisation, voire la modernité urbaine. » (Lord, 2006, p. 70)

Au début de ce mouvement de construction de Moncton comme « capitale culturelle » de l'Acadie (Boudreau, 2007; voir aussi Brun del Re, 2012), on retrouve le principe d'un choc des générations qui se décline en dichotomies : la modernité contre la tradition, bien sûr, qui rejoint l'opposition de la poésie des jeunes écrivains des années 1970 au roman d'Antonine Maillet, mais aussi l'urbanité s'affirmant contre la ruralité. Comme l'explique Raoul Boudreau, «Moncton incarnera donc l'urbanité comme lieu de fabrication de la modernité opposée à la ruralité comme lieu de sauvegarde et d'expression de la tradition. [...] Mise à distance des sources rurales n'égale pas reniement, mais passage à une autre étape. » (Boudreau, 2007, p. 44)

La création du mythe de Moncton comme espace conjuguant urbanité et modernité connaîtra déjà un premier « relais »(Brun del Re, 2012, p. 37) à l'intérieur de la même génération que Gérald Leblanc, mais dans le genre romanesque, d'abord chez Jean Babineau (Leclerc, 2005) puis chez France Daigle : « la ville romanesque de Daigle se fond à la ville poétique de Leblanc»(Brun del Re, 2012, p. 38; voir aussi Doyon-Gosselin et Morency, 2004). L'adhésion totale au mythe, encore plus que la poursuite de sa construction, se fait cependant sentir en particulier chez la génération d'artistes suivant Leblanc, celle de l'école Aberdeen, sauf que l'expression littéraire moderne que permet Moncton, de collective qu'elle était chez leur mentor, prend une connotation plus individuelle chez ces poètes : «En 1990, en 2000, le sujet poétique n'endosse plus cette conscience du “nous", mais simplement - pour autant que le terme soit bien choisi - un malaise existentiel, une peur d'être, ou plus 
PÉNÉLOPE CORMIER, « Le Manifeste scalène de la “ quatrième génération ” d'artiste en Acadie : vers une relève régionale »

exactement de ne pas être, déliée de tout fondement communautaire et historique, au profit d'une introspection forcenée. » (Laparra, 2004, p. 144)

Le succès monumental de cette entreprise de raccordement, dans les esprits et dans les faits, de l'urbanité monctonienne avec la modernité littéraire acadienne, menée et consolidée des années 1970 aux années 2000, ne doit cependant pas faire oublier les voix ou les phénomènes qui y résistent quelque peu, déjà par des contemporains de Leblanc. Pour revenir à Chiasson, il rappellera que la plupart des artistes acadiens de Moncton sont en fait originaires d'ailleurs en Acadie : «Même si cette ville est devenue, par la force de son infrastructure, une dimension importante de notre présence, il faut toutefois garder à l'esprit qu'elle s'alimente à tout un arrière-pays qui lui donne son envergure et son importance. » (2007, p. 14) Par ailleurs, dans sa thèse de doctorat mettant en relief la polarisation entre la ruralité et l'urbanité de la littérature acadienne, Andrée Mélissa Ferron présente le projet de Claude LeBouthillier comme porte-à-faux à celui de Leblanc, puisque

l'œuvre de Claude LeBouthillier aura réitéré l'adéquation entre acadianité et espace rural, et ce jusque dans le $21^{\mathrm{e}}$ siècle. Elle s'érige à partir d'un fond idéologique que nous nommons ruralisme, entendu ici non pas simplement dans son sens d'aménagement de l'espace rural [...], mais aussi dans sa part d'agrarisme, c'est-àdire dans l'opposition à la dénaturation de l'espace rural par l'industrialisation massive et l'idéologie capitaliste, dans la préconisation du retour à la terre, et dans la dénonciation de l'exode rural vers la ville. (2014, p. 254)

D’une part, la ruralité dont LeBouthillier se fait le porte-flambeau n'est pas d'inspiration ou d'aspiration moderne; d'autre part, Chiasson n'hésitera jamais, malgré sa méfiance envers Moncton, à reconnaître son ascendant moderne. Mais dans l'immédiat de l'an 2000, un nouveau phénomène en littérature acadienne bouleverse les termes : il s'agit de l'arrivée sur la scène littéraire acadienne d'artistes pour la plupart issus d'une nouvelle génération, certes, 
mais, plus radicalement, de la Nouvelle-Écosse. Ils proposent une délocalisation de Moncton (et du Nouveau-Brunswick), tout en se réclamant de la modernité.

\section{Sortie de Moncton 1 : De « Se sortir du bois... »à \#clareestsurlaboom}

En 2000, un numéro entier d'éloizes : la revue acadienne de création - basée à Moncton et alors dirigée par des poètes de l'école Aberdeen - est consacré aux artistes de la Nouvelle-Écosse qui, jusque-là, signaient principalement des textes dans Feux châlins, la revue de création de cette province. Le titre du numéro, «Se sortir du bois... », n'invitait peut-être pas d'emblée à remettre en question la répartition des compétences littéraires modernes et traditionnelles aux espaces urbains et ruraux respectivement. C'est en tout cas la lecture qu'on a pu en faire, en réaction à ce numéro :

Les écrivains qui habitent Moncton (qu'ils soient ou non originaires du Sud-Est de la province), ayant adopté cette ville comme lieu de vie, l'ont adoptée aussi comme espace d'écriture. En cela, leur attitude est bien différente de celles des jeunes écrivains acadiens de la Nouvelle-Écosse, par exemple, qui proposent des thématiques plus rurales (Cossette et Laparra, 2001, p. 146).

Cependant, il faut bien reconnaître que même avec cette mise à distance, l'accueil des artistes acadiens néo-écossais par une institution de Moncton a favorisé l'affirmation d'un mouvement qui s'est appuyé - temporairement, comme on le verra - sur le capital moderne de l'urbanité monctonienne pour procéder à une modernisation de l'espace rural acadien.

En effet, Georgette LeBlanc, en dirigeant ce numéro de la revue éloizes, amorçait un parcours qui l'a vue être publiée aux Éditions Perce-Neige à Moncton pour une première fois en 2006, recevoir le prix québécois Félix-Leclerc de la poésie en 2007, être finaliste pour un prix du Gouverneur général en 2014 et être nommée poète officielle du Parlement du Canada en 2018. Dans son texte de présentation du numéro, elle posait déjà, pour l'ensemble de ses 
PÉNÉLOPE CORMIER, «Le Manifeste scalène de la “ quatrième génération " d'artiste en Acadie : vers une relève régionale »

concitoyens, le défi de la création acadienne néo-écossaise en termes apparemment paradoxaux d'ouverture au monde et de maintien d'une authenticité : «En Nouvelle-Écosse, le bois nous a permis de garder une langue, des coutumes difficilement préservées dans une réalité urbaine et anglophone. Il faut peut-être se sortir du bois pour enfin comprendre que dans les bois les sources coulent toujours. » (LeBlanc, 2000, p. 6) Dans son article sur Alma, le premier recueil de LeBlanc, Jimmy Thibeault identifie à l'auteure néo-écossaise plus de liens avec les poètes acadiens urbains et modernes qui lui sont contemporains que les écritures de la ruralité des générations précédentes, allant jusqu'à faire exploser le parallélisme des dichotomies spatiales (ruralité-urbanité) et temporelles (traditionmodernité) : «Georgette LeBlanc démontre bien que l'espace rural s'est aussi mis au diapason du monde moderne » $(2016$, p. 143).

Avec Georgette LeBlanc, c'est au groupe de rap/hip hop Jacobus et Maleco, de la Baie Sainte-Marie en Nouvelle-Écosse, que 1'on doit, toujours au début des années 2000, la correspondance de la modernité et de la ruralité dans les arts acadiens. Encore là, l'accueil monctonien des propositions esthétiques modernes du groupe est essentiel au mouvement de balancier, comme l'explique en entrevue Alexandre Bilodeau, l'un des fondateurs du groupe : «so ça a décollé pas mal // effortless là // mais c'était direct le moment qu'on a quitté Clare [correspondant à la Baie Sainte-Marie] que ça a décollé // pis là // le monde de Clare euh // c'était dur de le faire décoller dans Clare mais là quand ça décollé à Moncton le monde de Clare aimait ça ${ }^{3} »$. Refondu en Radio Radio quelques années plus tard, le groupe

\footnotetext{
${ }^{3}$ Entrevue réalisée en 2015 dans le cadre du projet de recherche en littérature, sociolinguistique et traductologie Langue(s) et production culturelle depuis 2000 : construction discursive de la variété linguistique autour des arts en Acadie, dirigé par Mélanie LeBlanc et regroupant Annette Boudreau, Pénélope Cormier, Catherine
} 
a reproduit son succès extérieur en misant encore sur la modernité de ses propositions, cette fois sur la scène québécoise, comme l'analyse Catherine Leclerc : l'album « Belmundo regal [en 2010] poursuit le travail de positionnement du groupe sur un circuit international. Il décrit sa trajectoire d'un passé plus rural vers des zones urbaines, à partir d'une diversité d'expériences qui correspondent aux habitudes de vie cosmopolites et branchées. » (2016, p. 116)

Radio Radio joue sur les mêmes tableaux d'authenticité et de modernité que Georgette LeBlanc; en fait, la langue vernaculaire de la Baie Sainte-Marie - l'acadjonne que ces artistes mettent de l'avant dans leurs œuvres est contextualisée (géographiquement et historiquement) dans leur communauté d'origine, mais capitalise également sur la valorisation culturelle de l'hybridité linguistique dans un contexte mondialisé. Comme l’explique Mireille McLaughlin, les artistes de Radio Radio

mobilize the myth of marginality and oppression to showcase their upward social mobility and the new position of power in a globalized world. In their songs and videos, the rappers play with traditional images of oppression and sell Acadian rural coastal regions as an integral part of global « hip ». They are hip precisely because they are peripheral. (McLaughlin, 2013, p. 48)

Le risque de cette stratégie, bien sûr, est que l'affirmation rurale se fasse uniquement par la réactivation d'un stéréotype et donc le renvoi de la périphérie à un exotisme folklorique (voir McLaughlin, 2013 et Leclerc, 2016). Revenant sur la réception québécoise de Radio Radio, Leclerc indique que, dans une large mesure, le piège a été évité : « Ce qui était perçu comme un signe de faiblesse de la part des minorités francophones - la proximité avec l'anglais -

Leclerc, Nicole Nolette et Hubert Noël. Il a été financé de 2015 à 2017 par le Centre canadien de recherche sur les francophonies en milieu minoritaire (Université de Regina). 
PÉNÉLOPE CORMIER, « Le Manifeste scalène de la “ quatrième génération ” d'artiste en Acadie : vers une relève régionale »

place une portion de ces mêmes minorités en position de force par rapport aux Montréalais francophones eux aussi en situation de contact. Dans ce nouveau cadre, Radio Radio se trouve soudain à l'avant-garde! » $(2016$, p. 120)

À partir de ce revirement spectaculaire réalisé par Georgette LeBlanc et Radio Radio, ruralité peut signifier modernité en Acadie. Depuis, le succès des artistes issus de la Baie Sainte-Marie ne montre pas de signe de ralentissement. Le retour en « région » d'Alexandre Bilodeau (qui adopte à ce moment le nom d'artiste Arthur Comeau), et sa création de la Tide School, une maison de production de disque, a même fait exploser le nombre d'artistes à la Baie Sainte-Marie, alors que son réseau artistique, héritage de ses années avec Radio Radio, lui permet de multiplier les collaborations nationales et internationales. Dans un texte expliquant comment et pourquoi «Clare est sur la boom », la ressortissante néo-écossaise Céleste Godin situe ainsi le rôle de Bilodeau/Comeau :

Ces artistes flottent dans l'espace entre le très local (ils s'expriment dans le parler de la Baie, l'acadjonne, et souvent sur des moments de la vie dans cette région) et le global. C'est du local-planétaire. [...] En quittant Radio Radio et Montréal pour s'établir à Meteghan, [Arthur Comeau] montre que c'est possible de créer le son qu'on veut à partir de n'importe où et que nos communautés ne sont pas moins fécondes ni moins porteuses que Montréal. (2015, en ligne)

C'est donc toute une «nouvelle vague d'artistes » (Godin, 2015, en ligne) acadiens de la Nouvelle-Écosse qui ne sentent plus la nécessité de «sortir du bois » pour aspirer à la modernité artistique.

\section{Sortie de Moncton 2 : Pour une littérature du Nord}

En rendant visible l'existence d'une littérature acadienne se faisant dans le nord du NouveauBrunswick, le Manifeste scalène de Sébastien Bérubé, Gabriel Robichaud et Jonathan Roy

prend appui tant sur les stratégies des artistes acadiens pour construire Moncton comme 
capitale artistique urbaine et moderne que sur celles ayant établit la Baie Sainte-Marie comme un autre pôle de création acadien, rural et moderne cette fois. D'une part, il s'agit encore d'écrire les réalités acadiennes et le spectacle les évoque sans détour, par des références à l'histoire, à la littérature ou à la musique, ainsi que par des commentaires sur l'actualité sociale. D'autre part, et paradoxalement peut-être, c'est en renforçant les éléments de continuité de leur projet poétique par rapport à celui des écrivains acadiens qui les ont précédés que Bérubé, Robichaud et Roy procèdent à une affirmation générationnelle, dont l'originalité repose sur une affirmation littéraire régionale en Acadie.

Il revient à Andrée Mélissa Ferron d'avoir, la première, affirmé l'émergence de cette littérature acadienne du nord du Nouveau-Brunswick, dans un article de 2016 consacré à Apprendre à tomber (2012), le premier recueil de Roy. Formant ainsi un premier retour sur ce phénomène, avec certaines des critiques des œuvres de la relève (Doyon-Gosselin, 2017a et 2017b; Ferron 2016b; Kirouac Massicotte 2017), cet article en conceptualise deux enjeux fondamentaux : d'une part, la continuité intertextuelle; d'autre part, la rupture régionale. Pour commencer, il est vrai qu'avec les écrivains du Manifeste scalène, et avec ce spectacle dont l'objectif est justement de se définir comme écrivains acadiens, on se retrouve avant tout devant «une génération de poètes jetant un nouveau regard sur les textes dont ils sont les héritiers »(Ferron, 2016a, p. 155). En cela, ils suivent une tradition littéraire acadienne établie par Gérald Leblanc, dont la pratique intertextuelle « fera école et incitera les nouvelles générations d'écrivains - poètes surtout - qui émergeront des années 1980, 1990 et 2000 à la transtextualité endogène. » (Ferron, 2016a, p. 151)

Certains observateurs, cependant, n'ont vu qu'un automatisme dans cette conscience textuelle des prédécesseurs affichée par les nouveaux arrivants sur la scène littéraire 
PÉNÉLOPE CORMIER, « Le Manifeste scalène de la “ quatrième génération ” d'artiste en Acadie : vers une relève régionale »

acadienne, qu'une reprise non critique d'une propension de longue date en Acadie. En particulier, Mathieu Wade, dans sa critique du recueil Acadie Road (2018) de Gabriel Robichaud - dans lequel figure la contribution de Robichaud au Manifeste scalène - conteste l'utilité du

namedropping, quasiment érigé au rang de forme poétique. Quasiment. [...] C'est Gérald Leblanc, namedropper notoire, qui a lancé le bal alors que la littérature acadienne s'institutionnalisait. Chez lui, ça servait à élargir les horizons culturels de l'Acadie, faire entrer la Beat Generation, le jazz, la poésie française dans le giron culturel acadien, faire éclater les cadres culturels jusqu'alors admis. Ça servait aussi à fonder une communauté littéraire acadienne naissante en citant ses pairs. Chez Robichaud, c'est un peu l'inverse; ça sert à se replier sur le milieu artistique actuel, sur une francophonie établie. La démarche avait un sens dans les années 1970-80, mais elle perd de sa pertinence 40 ans plus tard. $(2019$, s. p.)

Les deux fonctions de la nomination - l'importation de références culturelles et l'établissement d'une communauté artistique locale - dans la poésie de Gérald Leblanc que reprend ici Wade sont évidemment les deux versants permettant la construction d'un capital littéraire en Acadie. Avec l'écriture de l'espace, qui fonctionne également sur le mode de la nomination chez Leblanc, cela concourt, comme on l'a vu, à l'établissement de Moncton comme centre littéraire en Acadie (voir Boudreau, 2007; Boudreau et White, 2009; Paré, 2007). Or, si la nomination - d'artistes ou de lieux - est bel et bien une technique d'écriture utilisée par Bérubé, Robichaud et Roy, elle répond à une autre logique, voire à une autre nécessité. En ce sens, il me semble approprié d'examiner plus longuement l'usage spécifique que peuvent faire les auteurs actuels des « codes de la littérature acadienne depuis les années $1970 »($ Wade, 2019, s. p.).

Certes, l'emploi d'une technique prouvée et fétiche de la littérature acadienne par des nouvelles plumes peut gêner à leur proclamation d'une originalité par rapport aux générations 
précédentes. Il faut voir toutefois qu'il s'agit du meilleur moyen pour un nouvel auteur d'intégrer une institution littéraire : par la démonstration, justement, que l'on en maîtrise les « codes ». C'est même l'une des conditions d'accès à l'existence littéraire, en Acadie comme ailleurs. Comme l'explique Ferron, l'intertextualité est une «stratégie de cohésion comme pour revendiquer une appartenance cénaculaire dans le contexte d'une institution littéraire née dans la marginalité et la double périphérie. » (2016a, p. 151) On trouve ici un écho à l'article de Marcel Olscamp sur la «convivialité » des espaces littéraires évoluant en dehors des grands centres, dans lequel il observe « un étonnant dispositif de salutations, de citations et de clins d'œil parfois un peu mystérieux pour un non-initié. Ce phénomène est particulièrement évident en Acadie » (2000, p. 497). Il est incontestable que les auteurs du Manifeste scalène veulent s'inscrire dans l'histoire littéraire acadienne.

Les références littéraires du Manifeste scalène sont cependant, à la différence de chez Leblanc par exemple, souvent des références au second degré, qui permettent à l'ironie envers les «maîtres» de cohabiter habilement avec l'hommage, en diluant sa cible (potentielle). Ainsi, dans les vers « À cause que c'est pas Antonine Maillet qui a fait la Sagouine / C'est plutôt la Sagouine qui a fait Antonine Maillet / Une voix Goncourt que personne a jamais lue » (2017, s. p.), on aurait du mal à identifier exactement l'objet de l'ironie : la domination de Maillet en littérature acadienne? la longévité d'une carrière qui serait portée, au final, par un seul succès populaire ? le renversement d'un personnage de théâtre en représentant exotique de toute une population ? l'importance démesurée accordée aux prix comme mesure de qualité littéraire ? la tendance à valoriser la personnalité publique plutôt que l'œuvre ? toutes ces réponses ? aucune ? Comme autre exemple de la portée de cette stratégie, mentionnons le vers autonome de Bérubé, «1755 qui jase avec une 
PÉNÉLOPE CORMIER, « Le Manifeste scalène de la “ quatrième génération ” d'artiste en Acadie : vers une relève régionale »

grenouille». Dans ce vers présenté sans plus de contexte, la charge ironique - ou son absence - demeure à la discrétion du lecteur/du spectateur. Décrie-t-on cyniquement la transformation d'un monument de la musique acadienne en animateur de foule d'enfants, ou salue-t-on plutôt son engagement envers la jeunesse acadienne ?

Outre l'ambiguité sémantique et le double jeu ironique que laissent planer la plupart des vers du Manifeste scalène renvoyant aux générations précédentes d'artistes acadiens, il s'agit pour les poètes de mettre à jour la «liste » des références littéraires et artistiques acadiennes de leurs prédécesseurs, ce qui a également l'avantage de procéder à la construction d'une cohésion de génération. C'est en ce sens que l'on peut interpréter les renvois apparemment gratuits et faciles aux groupes de musique Les Hôtesses d'Hilaire et Les Hay Babies, ainsi qu'à l'auteure-compositeure-interprète Lisa LeBlanc, dans les vers suivants : «À cause que nos hôtesses sont d'Hilaire / Une voix néguac and back / À cause qu'on trouve la beauté dans les champs de foins / Une voix ligne d'hydro » (2017, s. p.). Il faut également noter, ici, l'action d'une intertextualité «interne », où les vers de Robichaud et de Roy s'interpellent directement, s'alimentant aux mêmes sources.

Dans l'ensemble, le principal renvoi du spectacle relève de sa facture, puisque les textes de Bérubé, Robichaud et Roy se renvoient avant tout la balle entre eux. D'une part, la parenté de construction de leurs poèmes, sur le principe de l'anaphore, permet d'identifier les poètes : les vers « J'ai des... des... » sont de Bérubé, ceux qui répètent « À cause de... » en début de vers sont de Robichaud (et de Jean-Philippe Raîche, co-auteur du poème), alors que les vers évoquant «Une voix... une voix... » sont de Roy. D’autre part, la cohésion de l'ensemble, marquée par l'usage de l'anaphore, est renforcée par le mélange des textes, en particulier aux quelques endroits où les poètes lisent les vers des autres. L'un de ces passages, 
situé presque à la fin du spectacle, effectue la transition de l'inscription réflexive des poètes dans la continuité de la lignée des écrivains acadiens à l'affirmation de leur originalité par rapport à ce groupe, dont il est évident qu'elle passe par une proposition spatiale novatrice en littérature acadienne :

À cause qu'on connaît mieux nos ancêtres que nos descendants

À cause qu'ils nous ont nommés

À cause qu'ils nous ont écrits

À cause qu'on les a lus

À cause qu'on y croit

À cause qu'on en a fait notre voix

Une voix rurale

Une voix urbaine

Une voix que j'aime des fois

À cause que Caraquet s'est autoproclamée capitale

À cause que Moncton n'existe pas toute seule

À cause qu'Edmundston fait aussi partie du décor (2017, s. p.)

Naturellement, les trois derniers vers sont déclamés respectivement par Roy, Robichaud et Bérubé, en tant que représentants des villes nommées. C'est donc à la fin du Manifeste scalène que les trois poètes revendiquent comme proposition artistique l'enjeu spatial annoncé par le titre du spectacle, et se situant entre la dichotomie urbanité/ruralité en littérature acadienne et la triangularisation entre le nord-ouest, le nord-est et le sud-est de l'Acadie du Nouveau-Brunswick.

On en arrive donc au second enjeu d'une littérature acadienne du nord du NouveauBrunswick identifié par Andrée Mélissa Ferron : l'écriture de l'espace rural. Ce processus va de pair avec le phénomène d'(auto)référentialité littéraire que l'on vient d'examiner, car il relève pour Ferron de « l'implication des textes littéraires dans la construction de l'espace acadien » (2016a, p. 147). Bien sûr, cette littérarisation de l'espace est au cœur même de la 
PÉNÉLOPE CORMIER, « Le Manifeste scalène de la “ quatrième génération ” d'artiste en Acadie : vers une relève régionale »

construction de Moncton comme capitale littéraire acadienne: «l'espace monctonien comme un texte collectif » (Ferron, 2016a, p. 153). Or, dans son article sur le premier recueil de Roy, où elle s'attarde à la « double appartenance du poète au rural et à l'urbain (ou selon l'esprit néo-brunswickois, au "nord" et au "sud") » (2016a, p. 154), Ferron dégage le fossé de représentation littéraire des espaces urbains et ruraux en Acadie, du Sud et du Nord, de Moncton et de Caraquet (ou Edmundston dans le cas de Bérubé). Incontestablement, « [m]is en parallèle avec l'univers hypertrophié du Moncton littéraire, l'espace rural prend les contours du no man's land.» (Ferron, 2016a, p. 167) Les œuvres individuelles de Roy et de Bérubé construisent certes une carte littéraire de la Péninsule acadienne et du Madawaska, entre les « routes rurales» (Roy, 2012, p. 42) et « la boucane du moulin » (Bérubé, 2015, p. 11). La réception critique de leurs œuvres - en l'occurrence du recueil Là où les chemins de terre finissent (2017) de Bérubé - a d'ailleurs relevé la «volonté d'inscrire le bois et le Nord dans la littérature acadienne, mais aussi plus généralement dans l'imaginaire social de l'Acadie » (Kirouac Massicotte, 2017, s. p.; voir aussi Doyon-Gosselin, 2017a).

Dans son compte rendu de la première représentation du Manifeste scalène, Ferron situe ce travail dans la foulée de celui qui a permis d'inscrire Moncton sur la carte littéraire acadienne : «Le regard qu'auront promené Roy et Bérubé sur leurs “backyards” respectives, à l'instar de celui qu'avait posé Guy Arsenault sur son espace quelques décennies auparavant, aura justement témoigné d'une Acadie rurale crue, quotidienne» (2016, s. p.). En effet, le spectacle aborde directement les thèmes de la ruralité. Dès les premiers vers du spectacle, prononcés par Roy, le ton est donné : «Quand les buildings s'arrêtent / Entendre les voix qui s'empilent » (2017, s. p.). D'emblée, on se projette hors urbanité et la contribution du poète s'attachera justement à décliner la multiplicité des « voix » acadiennes, y compris rurales. 
Mais avant, Robichaud procède à un rappel historique signalant l'appartenance collective : «À cause qu'on voulait pas de roi / À cause qu'on prenait pas les armes / On nous a donnés » (2017, s. p.). Dès la première intervention de Bérubé, le manifeste s'engagera résolument dans le commentaire social sur des enjeux contemporains : «J'ai des rivières qui se boudent / Des forêts brevetées en cachette / [...] / J'ai les plus grandes marées du monde / Une population stuck à marée basse » $(2017$, s. p.). Comme on le voit, la portée du manifeste n'est pas explicitement régionaliste, tant s'en faut: elle se réclame même de l'ensemble de l'Acadie, réunissant les rivières, les forêts et la mer dans la voix du poète du Nord-Ouest, mais elle se préoccupe des aspects ruraux de l'Acadie, et en particulier de ses réalités difficiles. On est loin de l'évocation d'un exotisme comme la ruralité acadienne a souvent été sollicitée. Comme le rappelle fort à propos Ferron, «[t]oute fiction, toute magie semblent avoir quitté ces lieux: on y retrouve l'envers du théâtre que consistait la "joie de vivre" chronique, l'imaginaire pastoral et l'harmonie champêtre du récit originel acadien. » (2016a, p. 167) Dans le Manifeste scalène, Roy le confirme d'ailleurs aussitôt : il s'agit bel et bien de prendre le pouls d'« [u]ne voix nord vidée de son souffle » $(2017$, s. p.).

L'écriture du Nord est ainsi engagée, solidaire de la situation sociale précaire des plus démunis des régions acadiennes rurales, dont les problèmes tournent comme il se doit autour de l'exploitation des ressources naturelles et des enjeux économiques qui lui sont associés :

Une voix bitumineuse enlisée dans les dettes Une voix Irving fourrée à blanc

Une voix matière première that's it that's all

J'ai un voleur comme employeur majoritaire

Des épinettes bien alignés

Des terres de la couronne barrées par the Green Giant (2017, s. p.) 
PÉNÉLOPE CORMIER, «Le Manifeste scalène de la “ quatrième génération " d'artiste en Acadie : vers une relève régionale »

Comme Isabelle Kirouac Massicotte le notait par rapport au recueil là où les chemins de terre finissent, il s'agit d'une «vive critique des dérives capitalistes ainsi que de la perte des valeurs traditionnelles » $(2017$, s. p.) qui mène à la tension entre le milieu social traditionnel et l'aspiration artistique moderne. La section du Manifeste scalène où ce traditionalisme est le plus frappant est le développement sur la religion. Il peut sembler assez curieux, déjà, de voir surgir ce sujet de la plume de la nouvelle génération, mais Roy donne effectivement place à « une voix chrétienne » et à « une voix à genoux devant la bonne sainte anne » (2017, s. p.), tandis que Robichaud mentionne au détour d'un vers « qu'on va pu à l'église, mais qu'on garde nos clochers pour la guerre » (2017, s. p.). Pour sa part, Bérubé évoque «Des écoles vides comme des bancs d'église » $(2017$, s. p.) et aborde même directement la contradiction entre tradition et modernité dans l'espace rural en forçant une apposition : « J'ai du linge de première communion / Et des chandails de Metallica étendus sur la même corde » (2017, s. p.). Kirouac Massicotte a d'ailleurs noté la contradiction intrinsèque des références d'un tel «plaidoyer en faveur du rassemblement communautaire comme l'indique l'image ambiguë de l'église [...], qui signifie tout à la fois la vie en communauté et la tradition (connotées positivement par le poète), mais également la soumission et le dogmatisme. » (2017, s. p.)

Si la solidarité avec le milieu social est effectivement une caractéristique de l'écriture du Nord, et passe dans une certaine mesure par l'accréditation de ses éléments traditionnels, on note néanmoins une irrévérence déclarée envers la religion dans leur écriture, ainsi que leur refus de la soumission, qui passe chez Bérubé et Roy par la même image : «Le pignon de l'église a quelque chose d'attendrissant / Quand on s'arrête pour le regarder / [...] / Sa droiture n'a d'égal que les genoux qu'il a usés» (Bérubé, 2017, p. 24) et «l'usure 
correspondait parfaitement / à celle du prie-dieu retrouvé / vandalisé / dans sa chapelle » (Roy, 2012, p. 55). Il faut également rappeler que, si la littérature acadienne a su faire croire à la modernité de Moncton comme «Acadie City» (Paré, 1998), il n'en demeure que l'univers rural, notamment la tradition et la religion, hante aussi les écrits de Gérald Leblanc et de Guy Arsenault, les plus éminents «poètes de Moncton », comme le remarque François Paré dans Acadie Rock : «L'univers de Guy Arsenault est résolument celui de la campagne : c'est encore, au cœur de la ville, le village, avec ses rituels religieux et sa convivialité obsessive » (1998, p. 29).

Pour ce qui est, malgré tout, de faire entrer la modernité dans la ruralité, le poème «les poètes automatistes survivent mal en forêt» (2017, s. p.) de Roy procède à un renversement habile et stratégique de l'étrangeté. Si ruralité et modernité ne font pas bon ménage, on comprend que c'est la modernité, déracinée, qui est mal adaptée à la ruralité : «même épormyable l'orignal / mythomane tombe / sous la décharge du chasseur » (2012, p. 36). Bérubé prend la suggestion au rebond en proposant que la poésie n'est pas nécessairement assujettie aux codes de la modernité artistique : «Le plus grand des poètes / Que je connaisse / Ne savait pas lire / Mais limait une chainsaw / Comme personne » (2017, p. 55). En cela, les deux poètes rejoignent la promesse qu'Olscamp reconnaissait dans les milieux excentrés, celle d'« une littérature accessible, au sens le plus noble du terme; on découvre une parole neuve, surgissant comme une source d'un enthousiasme jamais démenti. » (2000, p. 507)

Le Manifeste scalène se solde par les mots « On crée » (2017, s. p.), clamés en chœur, une conclusion à la fois banale et fondamentale. Si le processus de résolution de la tension entre ruralité et modernité n'est certes pas complété, le spectacle laisse entrevoir la possibilité 
PÉNÉLOPE CORMIER, « Le Manifeste scalène de la “ quatrième génération ” d'artiste en Acadie : vers une relève régionale »

du développement d'une littérature acadienne moderne dans le Nord du Nouveau-Brunswick, dans ses espaces ruraux, ou pourrait-on dire dans ses petites villes, que sont Caraquet et Edmundston. Rappelons à ce propos le mot de Pierre Nepveu dans son essai Intérieurs $d u$ Nouveau Monde : «peut-on écrire les petites villes d'Amérique ?» (1998, p. 270). Et quand il pense à la petite ville comme espace hostile à l'art, ce n'est même pas à Caraquet ou Edmundston qu'il pense, mais bien à Moncton comme représentante acadienne de cette figure de « lieu humain sans grand caractère, à la croisée des routes, un pur ici sans raison d'être sinon sa propre existence. [La petite ville américaine] émeut par cette ténacité fragile et insensée, en même temps qu'elle suggère l'éphémère, une disparition possible. » (1998, p. 268) À l'interrogation de Nepveu, le Manifeste scalène répond cependant avec défiance « On crée ». En ce sens, l'espace du nord du Nouveau-Brunswick, du Caraquet de Roy au Edmundston de Bérubé, n'est peut-être pas encore tout à fait « sorti du bois », ou mis sur la carte littéraire de l'Acadie, comme l'est la Baie Sainte-Marie, ni non plus du paradoxe entre ruralité et modernité, mais la littérature acadienne est certainement en voie de sortir de Moncton.

\section{«Edmundston is the place to be »}

Il se passe quelque chose dans le nord du Nouveau-Brunswick. L'une des chansons de l'album Viens avec moi (2018) des Hôtesses d'Hilaire, groupe de musique de la Péninsule acadienne, s'intitule «L'Acadie des terres et des forêts » et propose «Edmundston is the place to be » comme refrain, comme pour nous convaincre qu'il s'y passe effectivement quelque chose. Le chanteur Serge Brideau va même jusqu'à scander que «[ç]a fait du bien de sortir de la Péninsule » (2018, s. p.), ce qui pourrait être interprété comme une boutade - peu de gens vont considérer sortir de la Péninsule acadienne pour se rendre à Edmundston 
comme un signe de progrès -, si ce n'était le contexte d'émergence d'une production artistique rurale et moderne ancrée au Nouveau-Brunswick, présentée dans cet article à partir du spectacle poétique Manifeste scalène de Sébastien Bérubé, Gabriel Robichaud et Jonathan Roy. La meilleure preuve de la construction et de la consolidation de cette nouvelle régionalisation de la littérature acadienne est sans doute sa reconnaissance, transversale en quelque sorte, par les artistes acadiens eux-mêmes. D'ailleurs, en décembre 2018, le groupe Cy de la Baie Sainte-Marie (qui compte aussi un membre de Moncton) lançait la chanson «Les gens du Nord», une ode à la résilience des pêcheurs, pour annoncer la sortie d'un nouvel album au printemps 2019.

Bien sûr, il y a toujours eu des artistes dans le nord du Nouveau-Brunswick, et des artistes du Nord déménagés ailleurs, dont la contribution à l'histoire de l'art acadien est fondamentale. Force est de reconnaître, cependant, que l'on assiste présentement à un mouvement collectif, avec un nombre de plus en plus significatif d'artistes y participant, qui permet de penser que le phénomène d'affirmation d'une culture acadienne moderne s'exprimant depuis le nord du Nouveau-Brunswick ne va que s'accroître. D'autres œuvres récentes, comme les pièces de théâtre de Christian Essiambre (avec Philippe Soldevila, Les trois exils de Christian E., 2013) ou de Marcel-Romain Thériault (Le filet, 2009), contribuent à écrire le Nord. D'autres membres de la « quatrième génération » d'artistes acadiens, comme Joannie Thomas ou Monica Bolduc en poésie, en développant une écriture autrement engagée, en l'occurrence féministe, permettent d'espérer que l'écriture du Nord se diversifie.

Enfin, comme c'est le cas à la Baie Sainte-Marie, et comme on l'a vu avec l'exemple des Hôtesses d'Hilaire, les groupes de musique et les auteurs-compositeurs-interprètes participent du même mouvement que les écrivains, et le consolident par la même occasion. 
PÉNÉLOPE CORMIER, « Le Manifeste scalène de la “ quatrième génération ” d'artiste en Acadie : vers une relève régionale »

Ainsi en est-il des Hay Babies, qui affichent dans leur succès « Néguac and Back » la même tension entre tradition et modernité que les poètes du Manifeste scalène et qui est au centre de la créativité rurale du nord du Nouveau-Brunswick. Dans cette chanson sur l'exode rural, on mesure en effet la perte de la communauté par une référence religieuse : «Qui s'qui va sonner les cloches de l'église ?» (2014, s. p.) Pour sa part, Pascal Lejeune chante les lieux de la Péninsule acadienne depuis qu'il a adopté le nom d'artiste Thomé Young : «Enfin sur la 11 pour retourner chez nous / J'ai pris le chemin des Ressources / Pour retourner à la source / Après le croche à Edgar / C'est là qu'j' arrête mon char » (2014, s. p.). Ce passage est tiré de son album Victoria, au sujet duquel Roy a consacré un long poème-hommage, « sur un nowhere alentour de victoria », inclus dans son recueil savèches à fragmentation : «j'sais pas c'est l'affaire avec le nord / pis les disques de char» (2019, p. 105). Ce jeu de clins d'œil entre les artistes, qui a fait le succès de Moncton en littérature acadienne, est manifestement bien actif dans le Nord - et productif, puisqu'il semble mener à la poursuite du projet des précédentes générations artistiques, soit d'inscrire les réalités acadiennes dans les arts.

À ce sujet, il faut mentionner un autre passage-clé du Manifeste scalène, clamé en chœur par Sébastien Bérubé, Gabriel Robichaud et Jonathan Roy : «À cause que le poète, artiste visuel, dramaturge, cinéaste, réalisateur, ex-lieutenant-gouverneur et Chevalier de l'Ordre français des arts et des lettres Herménégilde Chiasson n'a pas encore tout fait » (2017, s. p.). Rappelons que pour Chiasson, le projet essentiel de la littérature acadienne est « de réconcilier Acadie et modernité » (1998b, p. 81), et que bien qu'il reconnaisse volontiers l'importance de Moncton pour la littérature acadienne, il ne voit pas d'un bon œil l'exclusivité, jusqu'à peu de temps passé, de la ville sur la modernité artistique en Acadie. En ce sens, les poètes du Manifeste scalène peuvent bien afficher une ironie à son égard, 
puisqu'ils poursuivent effectivement le travail incomplet de leurs prédécesseurs. Mais pour l'instant, dans le nord du Nouveau-Brunswick, il reste que « [c]henous est une job / jamais finie » (Roy, 2019, p.115), comme l'indique le titre de la section de savèches à fragmentation qui ne contient qu'un seul poème, une version étendue du poème « voix rurale » qui figure dans le Manifeste scalène.

En plus du caractère inachevé, ou encore en construction, de cette proposition artistique, il faut bien reconnaître, en terme de parcours, qu'une seule œuvre, même tripartite, et qu'un seul représentant par région, ne sont pas des raisons suffisantes pour tirer des conclusions collectives sur la relève en Acadie. Au niveau conceptuel, l'intérêt de leur contribution est de diversifier - et par le fait même nuancer - comment on envisage l'évolution de la littérature acadienne. En effet, une histoire littéraire acadienne se concevant comme une succession de "générations » artistiques et démographiques en perpétuelle relève exclut un certain nombre de trajectoires, et non des moindres - on pense au premier chef à Serge Patrice Thibodeau, qui publie sa première œuvre en 1990 au même moment que les premiers membres de l'école Aberdeen, mais qui n'en partage assurément pas l'esthétique. Pour sa part, Georgette LeBlanc commence à publier au milieu des années 2000, alors que l'école Aberdeen dont elle est contemporaine tire à sa fin, mais sa contribution s'interprète mieux si on l'envisage dans le cadre d'une relève régionale plutôt que générationnelle, dans le mouvement d'écriture d'une ruralité moderne en Acadie. En l'occurrence, les poètes du Manifeste scalène s'inscrivent dans une certaine mesure «en relève » à la fois de l'école Aberdeen, selon le principe générationnel, et des artistes de la Baie Sainte-Marie, selon le principe régional. Par ailleurs, ni l'une ni l'autre de ces perspectives ne permet encore d'intégrer de manière entièrement satisfaisante les œuvres non 
PÉNÉLOPE CORMIER, «Le Manifeste scalène de la " quatrième génération " d'artiste en Acadie : vers une relève régionale »

particularisantes; évoquons cette fois le cas de la dramaturge Emma Haché, installée dans la

Péninsule acadienne mais dont les pièces publiées sont décontextualisées. Il apparaît donc que chaque explication - générationnelle ou régionale - de l'histoire de la littérature acadienne entraîne son lot d'exclusions, d'« électrons libres ». Or, dans l'ensemble, cette impossible systématisation de la production littéraire acadienne est assurément signe de dynamisme et il est peut-être utile pour la critique d'envisager avec flexibilité une multiplicité de perspectives d'approche des relèves.

\section{Bibliographie}

BÉRUBÉ, Sébastien (2015), sous la boucane du moulin, Moncton, Perce-Neige, coll. «Poésie/Rafale».

BÉRUBÉ, Sébastien (2017), là où les chemins de terre finissent, Moncton, Perce-Neige, coll. «Poésie/Rafale ».

BÉRUBÉ, Sébastien, Gabriel RoBichaud et Jonathan Roy (2017), Manifeste scalène, inédit.

BOUDREAU, Raoul (2004), «L'écrivain et les honneurs : la nomination d'Herménégilde Chiasson au poste de lieutenant-gouverneur du N.-B. », Littérature canadienne / Canadian Literature, no 180, p. 189-194.

BOUdREAU, Raoul (2005), «Les poètes acadiens de la relève », Études en littérature canadienne/Studies in Canadian Literature, vol. 30, no 1, p. 211-225.

BOUDREAU, Raoul (2007), "La création de Moncton comme "capitale culturelle" dans l'œuvre de Gérald Leblanc », Revue de l'Université de Moncton, vol. 38, no 1, p. 33-56.

BOUDREAU, Raoul et Mylène WHITE (2009), « Gérald Leblanc : écrivain cartographe », dans Marie-Linda LORD et Denis BouRQue (dir.), Paysages imaginaires d'Acadie. Un atlas littéraire, Moncton, Institut d'études acadiennes et Chaire de recherche en études acadiennes, p. 43-55.

BRUCE, Clint (2005), « Gérald Leblanc et l'univers micro-cosmopolite de Moncton », Études canadiennes / Canadian Studies, no 58, p. 205-220. 
Brun Del Re, Ariane (2012), Portrait de villes littéraires : Moncton et Sudbury, thèse de maîtrise, Ottawa, Université d'Ottawa.

BRUn DEL RE, Ariane et Pénélope CORMIER (2015), «Actualité artistique et réception immédiate : les enjeux de la critique en Acadie », Port-Acadie, no 27, p. 27-46.

CHIASsON, Herménégilde (1994), «Le rôle de l'artiste dans la communauté acadienne », Revue de l'Université de Moncton, vol. 27, no 1, p. 317-330.

ChiAsson, Herménégilde (1998a), « La symbiose des formes artistiques », inédit.

CHIASsON, Herménégilde (1998b), « Traversée », Tangence, no 58, p. 77-92.

Chiasson, Herménégilde (2004), «Trajectoire et nostalgie », Neue Romania, no 29, p. 39-63.

Chiasson, Herménégilde (2005), «Considérations identitaires et culturelles sur l'Acadie moderne », Études canadiennes / Canadian Studies, no 58, p. 11-20.

ChIASsON, Herménégilde (2006), «L’Acadie pays sans frontière », inédit.

Chiasson, Herménégilde (2007), «Visions de Gérald », Revue de l'Université de Moncton, vol. 38, no 1, p. 7-31.

Chiasson, Herménégilde (2017), (12) abécédaires, Sudbury, Prise de parole.

CORMIER, Pénélope (2009), « Le passé, le présent et l'avenir de la littérature acadienne chez Herménégilde Chiasson », Voix et images, vol. 35, no 1, p. 51-62.

CORMIER, Pénélope (2012), « Les jeunes poètes à l'école Aberdeen : portrait institutionnel et littéraire », dans Jacques PAQUIN (dir.), Nouveaux territoires de la poésie francophone au Canada (1970-2000), Ottawa, Les Presses de l'Université d'Ottawa, « Archives des lettres canadiennes » Tome XV, p. 179-204.

COSSETTE, Isabelle et Manon LAPARRA (2001), «Les voix nocturnes: modes de représentation de la cité dans la poésie acadienne contemporaine », Francophonies d'Amérique, no 12, p. 145-152.

DoYON-Gosselin, Benoit (2005), «Y a-t-il une relève en poésie acadienne? Les nouveaux poètes acadiens à la croisée des chemins », Liaison, no 129, p. 38-40.

Doyon-Gosselin, Benoit (2015), «Pour une géocritique de Moncton », Cadernos de literatura comparada, no 33, p. 53-67.

DoyON-Gosselin, Benoit (2017a), «Là où la poésie commence », Liaison, no 176, p. 54. 
PÉNÉLOPE CORMIER, « Le Manifeste scalène de la “ quatrième génération ” d'artiste en Acadie : vers une relève régionale »

Doyon-Gosselin, Benoit (2017b, 23 août), «Loin d'être un cul-de-sac » (en ligne), Astheure, récupéré le 12 juin 2019 de : https://astheure.com/2017/08/23/loin-detre-un-culde-sac-benoit-doyon-gosselin/

Doyon-Gosselin, Benoit et Jean Morency (2004), « Le monde de Moncton, Moncton ville du monde. L'inscription de la ville dans les romans récents de France Daigle », Voix et Images, no 87, p. 69-83.

Essiambre, Christian et Philippe Soldevila (2013), Les trois exils de Christian E., Montréal, Dramaturges Éditeurs.

FERRON, Andrée Mélissa (2014), Écrire l'espace acadien : dialectique du rural et de l'urbain dans les auvres de Claude LeBouthillier et Gérald Leblanc, thèse de doctorat, Edmonton, Université de l'Alberta.

FERron, Andrée Mélissa (2016a), «Entre Moncton et Caraquet : le transit et le transitif chez Jonathan Roy », dans Cécilia W. FRANCIS et Robert VIAU (dir.), Littérature acadienne du $21^{e}$ siècle, Moncton, Perce-Neige, coll. « Archipel / APLAQA », p. 147-175.

FERron, Andrée Mélissa (2016b, 17 mai), « Manifeste Scalène : quand l'Acadie refuse d'être équilatérale » (en ligne), Astheure, récupéré le 26 mars 2019 de : https://astheure.com/2016/05/17/manifeste-scalene-quand-lacadie-refuse-detre-equilateraleandree-melissa-ferron/

GAUVIN, Karine (2004), «Une analyse discursive de l'identité acadienne à l'aube du Congrès mondial acadien », dans Denise DESHAIES et Diane VINCENT (dir.), Discours et constructions identitaires, Québec, Presses de l'Université Laval, p. 57-76.

Godin, Céleste (2015, 12 novembre), «L'onomatopée, le miroir et les alias : \#clareestsurlaboom » (en ligne), Astheure, récupéré le 26 mars 2019 de : https://astheure.com/2015/11/12/lonomatopee-le-miroir-et-les-alias-clareestsurlaboomceleste-godin/

Kirouac MAssicotTe, Isabelle (2017, 20 septembre), « Là où les chemins de terre finissent : un cri de terre du Nord» (en ligne), Astheure, récupéré le 26 mars 2019 de : https://astheure.com/2017/09/20/la-ou-les-chemins-de-terre-finissent-un-cri-de-terre-dunord-isabelle-kirouac-massicotte/

LAPARRA, Manon (2004), «Clamer le tabou : la poésie acadienne contemporaine ou la Revendication d'une marginalité systématique », Neue Romania, no 29, p. 135-148.

LEBLANC, Georgette (2000), «Avant-propos », éloizes : la revue acadienne de création, no 29 , p. 6. 
LECLERC, Catherine (2005), « De Bloop à Bloupe, de Moncton à Monckton. L'énonciation de la ville dans Bloupe de Jean Babineau », dans Adelaide Russo et Simon HAREL (dir.), Lieux propices. L'énonciation des lieux/Le lieu de l'énonciation dans les contextes francophones interculturels, Québec, Presses de l'Université Laval, p. 281-293.

LECLERC, Catherine (2010), Des langues en partage? Cohabitation du français et de l'anglais en littérature contemporaine, Montréal, XYZ.

LECLERC, Catherine (2016), « Radio Radio à Montréal : "la right side of the wrong" », Revue de l'Université de Moncton, vol. 47, no 2, p. 95-128.

Les Hay B ABIES (2014), Mon Homesick Heart (CD), Montréal, Simone Records.

Les Hôtesses D’Hilaire (2018), Viens avec moi (CD), Moncton, Studio B-12.

LORD, Marie-Linda (2006), "Identité et urbanité dans la littérature acadienne », dans Madeleine FRÉDÉRIC et Serge JAUMAIN (dir.), Regards croisés sur l'histoire et la littérature acadiennes, Bruxelles, P.I.E.-Peter Lang, p. 67-85.

MCLAughlin, Mireille (2001), «Les représentations linguistiques des jeunes écrivains du Sud-Est du Nouveau-Brunswick et leur impact sur la littérature acadienne », Francophonies d'Amérique, no 12, p. 133-143.

MCLAughlin, Mireille (2013), «What Makes Art Acadian? », dans Sari PIETIKÄINEN et Helen Kelly-Holmes (dir.), Multilingualism and the Periphery, Oxford, Oxford University Press, p. 35-54.

NEPVEu, Pierre (1998), Intérieurs du Nouveau Monde. Essais sur les littératures du Québec et des Amériques, Montréal, Boréal.

OlsCAMP, Marcel (2000), «Les poètes de la convivialité », dans Robert ViAU (dir.), La création littéraire dans le contexte de l'exiguïté, Québec, MNH, p. 495-507.

PARÉ, François (1998), «Acadie City ou l'invention de la ville », Tangence, no 58, p. 19-34.

PARÉ, François (2007), «Leblanc, Ginsberg, Hakim Bey et autres visionnaires », Revue de l'Université de Moncton, vol. 38, no 1, p. 75-92.

PILON, Jean-Guy (1969), « Journal de bord », Liberté, vol. 11, no 5, p. 154-163.

Robichaud, Gabriel (2018), Acadie Road, Moncton, Perce-Neige, coll. « Poésie ».

Roy, Jonathan (2012), Apprendre à tomber, Moncton, Perce-Neige, coll. « Poésie ». 
PÉNÉLOPE CORMIER, « Le Manifeste scalène de la “ quatrième génération ” d'artiste en Acadie : vers une relève régionale »

RoY, Jonathan (2014, 12 novembre), « voix rurale », dans Jonathan RoY, royjaune, récupéré le 13 mars 2019 de : https://royjaune.wordpress.com/2014/11/12/voix-rurale/

RoY, Jonathan (2019), savèches à fragmentation, Moncton, Perce-Neige, coll. « Poésie ».

THÉRIAUlt, Marcel-Romain (2009), Le filet : tragédie maritime, Sudbury, Prise de parole, coll. « Théâtre ».

ThiBeAult, Jimmy (2016), «Dire l'Acadie autrement: la reconfiguration des espaces identitaires acadiens dans la poésie récente », dans Jimmy THIBEAULT, Daniel LoNG, Désiré NYela et Jean WiLSON (dir.), Au-delà de l'exiguïté. Échos et convergences dans les littératures minoritaires, Moncton, Perce-Neige, coll. « Archipel / APLAQA », p. 119-144.

WADE, Mathieu (2019, 22 mars), «Acadie Road: droit dans un cul-de-sac » (en ligne), Astheure, récupéré le 26 mars 2019 de : https://astheure.com/2019/03/22/acadie-road-droitdans-un-cul-de-sac-mathieu-wade/

YounG, Thomé (2014), Victoria (CD), s. 1.

\title{
Résumé
}

Cet article examine le phénomène d'émergence récente d'une activité littéraire ancrée dans le nord du Nouveau-Brunswick. Au centre de l'analyse est le spectacle de poésie Manifeste scalène (2017) dont les auteurs, Sébastien Bérubé, Gabriel Robichaud et Jonathan Roy, se présentent comme des membres de la plus récente génération d'auteurs acadiens, mais surtout des représentants de chaque région - le Nord-Ouest, le Sud-Est et le Nord-Est respectivement - de l'Acadie du Nouveau-Brunswick. Il s'agit de replacer cette œuvre dans la quête de réconciliation de l'Acadie et de la modernité artistique qui est le fil directeur de l'histoire littéraire acadienne contemporaine. Après la construction de Moncton comme centre urbain de la modernité artistique acadienne, de 1970 à 2005, les artistes de la Baie Sainte-Marie ont revendiqué cette région de la Nouvelle-Écosse comme pôle de création rural et moderne au cours des années 2000. À leur suite, les poètes du Manifeste scalène procèdent à l'affirmation régionale artistique, moderne et rurale, du nord du Nouveau-Brunswick cette fois, appuyés par d'autres artistes, notamment en musique (Thomé Young, Les Hay Babies, Les Hôtesses d'Hilaire).

\begin{abstract}
This article examines the recent emergence of an Acadian literary practice in northern New Brunswick. The proposed analysis of the poetic performance Manifeste scalène (2017), by Sébastien Bérubé, Gabriel Robichaud, and Jonathan Roy, highlights the authors' identification not only as members of the newest generation of Acadian writers, but also as representatives of the different Acadian regions-respectively the North West, the South East and the North East-of the province. This in effect places them in continuity with the other periods of contemporary Acadian literature: first, the construction of Moncton as the urban
\end{abstract}


Acadian center of modernist art (1970-2005), and second, the emergence of the artists from the Nova Scotian region la Baie Sainte-Marie, bringing artistic modernity to a rural space (since 2005). The poets of the Manifeste scalène, as well as other regional artists (particularly musicians such as Thomé Young, Les Hay Babies and Les Hôtesses d'Hilaire), further this trend, posing northern New Brunswick as a place where modernity and rurality can cohabit.

\section{Notice biobibliographique}

Pénélope Cormier est professeure de littérature au campus d'Edmundston de l'Université de Moncton. Ses recherches portent sur les littératures acadiennes et franco-canadiennes selon une perspective de formalisme sociologique; son projet actuel est de faire une histoire de la littérature acadienne à partir de l'évolution de ses formes littéraires plutôt qu'à partir de la construction d'un récit national. Elle est membre du Groupe de recherche sur l'Acadie contemporaine (GRAC), du groupe de recherche Nouvelles solidarités en littérature francocanadienne, du Groupe de recherche interdisciplinaire sur les cultures en contact (GRICC) et du Groupe de recherche en édition critique de l'Université de Moncton (GRÉCUM). Depuis une quinzaine d'années, elle recense l'actualité artistique acadienne pour plusieurs périodiques culturels; elle est aussi cofondatrice et coéditrice de l'espace de critique artistique franco-canadienne du webzine Astheure. 